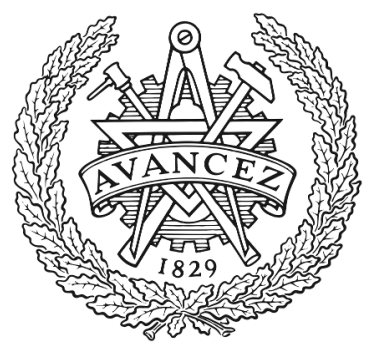

CHALMERS

UNIVERSITY OF TECHNOLOGY

\title{
Tunable Phases of Moiré Excitons in van der Waals Heterostructures
}

Downloaded from: https://research.chalmers.se, 2023-04-25 23:54 UTC

Citation for the original published paper (version of record):

Brem, S., Linderälv, C., Erhart, P. et al (2020). Tunable Phases of Moiré Excitons in van der Waals

Heterostructures. Nano Letters, 20(12): 8534-8540. http://dx.doi.org/10.1021/acs.nanolett.0c03019

N.B. When citing this work, cite the original published paper. 


\title{
Tunable Phases of Moiré Excitons in van der Waals Heterostructures
}

\author{
Samuel Brem, Christopher Linderälv, Paul Erhart, and Ermin Malic
}

Cite This: Nano Lett. 2020, 20, 8534-8540

Read Online

ABSTRACT: Stacking monolayers of transition metal dichalcogenides into a heterostructure with a finite twist-angle gives rise to artificial moiré superlattices with a tunable periodicity. As a consequence, excitons experience a periodic potential, which can be exploited to tailor optoelectronic properties of these materials. Whereas recent experimental studies have confirmed twistangle-dependent optical spectra, the microscopic origin of moire exciton resonances has not been fully clarified yet. Here, we combine first-principles calculations with the excitonic density matrix formalism to study transitions between different moire exciton phases and their impact on optical properties of the twisted $\mathrm{MoSe}_{2} / \mathrm{WSe}_{2}$ heterostructure. At angles smaller than $2^{\circ}$, we find flat, moiré-trapped states for inter- and intralayer excitons. This moiré exciton phase changes into completely delocalized states at $3^{\circ}$. We predict a linear and quadratic twist-angle dependence of excitonic resonances for the moire-trapped and delocalized exciton phases, respectively.

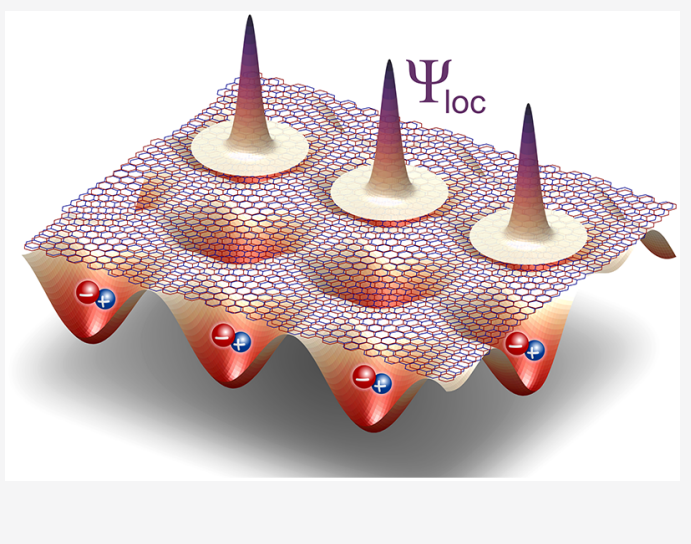

KEYWORDS: moiré excitons, twisted bilayer, van der Waals heterostructure, quantum emitter array, moire potential

A tomically thin quantum materials, such as graphene and transition metal dichalcogenides (TMDs), obtain their unique properties by hosting strongly interacting quasiparticles. In particular, the strong Coulomb interaction in TMDs allows the dynamics of excitons to be to studied. ${ }^{1-4}$ In a TMD monolayer, excitonic properties can be widely tuned through strain ${ }^{5,6}$ and dielectric engineering. ${ }^{7-9}$ Moreover, two monolayer TMDs can be vertically stacked to form a type-II heterostructure, giving rise to spatially indirect and therefore ultrastable interlayer excitons ${ }^{10-12}$ (Figure 1a). Recent studies $^{13-16}$ have shown that artificial moire superlattices can be created by vertically stacking monolayers with a finite twistangle, giving rise to a tunable modification of exciton features in optical spectra. Theoretical studies on homo- ${ }^{17}$ and resonantly aligned heterobilayers ${ }^{18}$ show that the twisttunability can be attributed to a stacking-dependent hybridization of intra- and interlayer excitons. ${ }^{19-21}$ Moreover, recent studies on heterostructures with a large band offset, where hybridization at the $\mathrm{K}$ point is negligible, suggest that moiré patterns lead to a spatially varying interlayer distance and an electronic band gap. ${ }^{22-24}$ Consequently, excitons experience a moiré periodic potential, which can be exploited to tailor exciton transport properties ${ }^{25}$ or even create tunable quantum emitter arrays. $^{26}$

To be able to exploit the technological potential and to design future experiments on van der Waals stacked superlattices, microscopic insights into moiré excitons are needed. First-principles computations can provide important insights about the electronic structure in these systems, ${ }^{27-31}$ but they are fundamentally limited to perfectly aligned or very specific twisted structures with relatively small supercells. However most exciting phenomena, such as superconductivity in magicangle bilayer graphene, ${ }^{32,33}$ are expected to occur at very small twist-angles with large supercells including thousands of atoms. Therefore, effective continuum models ${ }^{23,24}$ as used for bilayer graphene are needed to describe moiré excitons at small twistangles.

In this work, we combine first-principles calculations with the excitonic density matrix formalism to develop a materialspecific and realistic exciton model for small-angle twisted $\mathrm{MoSe}_{2} / \mathrm{WSe}_{2}$ heterostructures. Based on a microscopic approach, we calculate the band structure and wave functions of intra- and interlayer excitons within a twist-tunable moiré lattice as well as the resulting optical response of these compound particles. For a range of small twist-angles, we predict completely flat exciton bands for both intra- as well as interlayer excitons corresponding to moiré trapped, localized quantum emitters. However, we reveal that this moire exciton phase quickly changes with increasing twist-angle, and at $3^{\circ}$, there are only delocalized excitons (Figure $1 \mathrm{~b}$ ). We find the emergence of multiple moire exciton peaks in the absorption, whose spectral shifts with varying twist-angle are characteristic for the trapped or delocalized phase. Previous studies predicted

Received: July 22, 2020

Revised: September 24, 2020

Published: September 24, 2020 
a

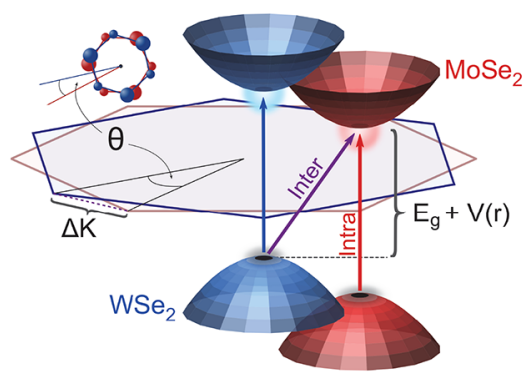

b

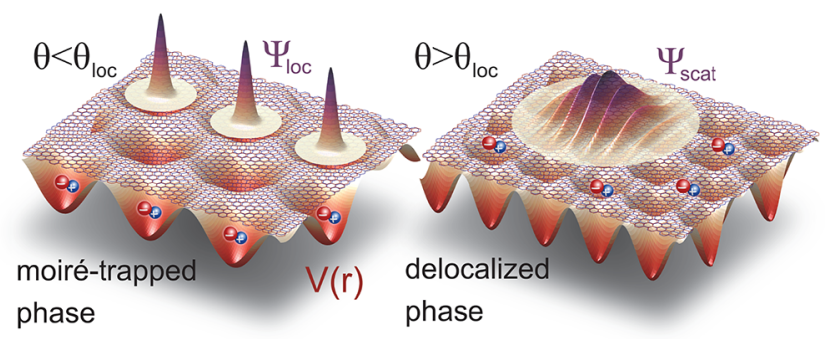

Figure 1. Schematic illustration of moiré excitons. (a) van der Waals heterostructures can host intra- (red and blue) as well as interlayer excitons (purple) at the $\mathrm{K}$ points (corners) of their hexagonal Brillouin zones. A finite stacking angle $\theta$ gives rise to a mismatch of the dispersions in momentum space and at the same time leads to a spatially periodic moiré potential $V(r)$. (b) Depending on the length of the moire period, the exciton center-of-mass motion can become quantized, leading to either moiré-trapped (left) or delocalized scattering states (right).

rather shallow moiré potentials for intralayer excitons. Here, we show that GW corrections of the electronic band structure lead to much larger intralayer band gap variations and a moiréinduced double peak structure characterizing intralayer excitons. Overall, our work provides microscopic insights and a comprehensive picture that unifies different moiré exciton phases and their optical signatures.

\section{RESULTS}

Moiré Potential. In this work, we study the type-II $\mathrm{MoSe}_{2} /$ $\mathrm{WSe}_{2}$ heterostructure, focusing on the energetically lowest interband transitions at the $\mathrm{K}$ points of the hexagonal Brillouin zone. First-principles calculations of the electronic structure in this material suggest that the wave functions at these band edges are strongly layer-polarized; ${ }^{29,30}$ that is, the monolayer eigenstates only weakly hybridize. However, recent experimental and computational studies suggest that the valence and conduction band energies at the $K$ point nonetheless significantly vary with the geometrical alignment of the two layers. $^{22,23,26}$ Consequently, in a twisted stacking configuration, where the local alignment of atoms is periodically changing, a spatially varying moiré potential is expected to emerge.

We model the moiré Hamilton operator by assuming that the energy variation $V$ results from interactions of the dorbitals (composing electronic states at the $\mathrm{K}$ point) with the effective atomic potentials of the neighboring layer (see Supporting Information):

$$
H=\sum_{\mathbf{k}, \mathbf{q}} V_{\mathbf{q}} a_{\mathbf{k}+\mathbf{q}}^{\dagger} a_{\mathbf{k}}+\text { h.c., } V_{\mathbf{q}}=v_{0} \sum_{n=0}^{2} e^{i \hat{\mathbf{G}}_{n} \mathbf{D}} \delta_{\mathbf{q}, \mathbf{g}_{n}}
$$

Here, $a_{\mathbf{k}}$ is the field operator of an electron with momentum $K$ $+\mathbf{k}$, which interacts with the moire potential determined by the lateral displacement of the two layers $\mathbf{D}$ and the fundamental translation $\mathbf{G}_{l}$ of the reciprocal lattice of layer $l$ with $\hat{\mathbf{G}}_{n}=C_{3}^{n}\left(\mathbf{G}_{1}+\mathbf{G}_{2}\right) / 2$ and $\mathbf{g}_{n}=C_{3}^{n}\left(\mathbf{G}_{2}-\mathbf{G}_{1}\right)$. The operator $\mathrm{C}_{3}$ rotates a vector by $120^{\circ}$, reflecting the hexagonal symmetry of the two subsystems. The form of eq 1 is equivalent to phenomenological formulas applied in previous studies, ${ }^{23,24,26}$ but it is derived here from a microscopic approach. When setting the twist-angle to zero, eq 1 collapses to a spatially independent energy shift. This shift, however, depends on the lateral displacement $\mathbf{D}$ that determines the atomic registry between the layers. Hence, the complex-valued parameter $v_{0}$ is obtained by comparing the band energies obtained by firstprinciple methods for different high-symmetry stackings. This allows the $\mathrm{ab}$ initio calculations to be incorporated into our effective continuum model. It is important to note that the continuum model is limited to small angles/large moiré periods and represents a harmonic interpolation between highsymmetry stackings within the superlattice. Twist-angledependent changes in the overall amplitude of the moiré potential (e.g., via emerging strain gradients) are not captured by the model.

The obtained energy shifts agree well with previously reported values for DFT $+\mathrm{GW}$ calculations, ${ }^{30}$ and the analytical model excellently reproduces the displacement dependence by fixing only two parameters per band (Figure 2a). The advantage of the developed microscopic model is that we can now use an analytical expression to model the moiré potential in an arbitrarily twisted material.

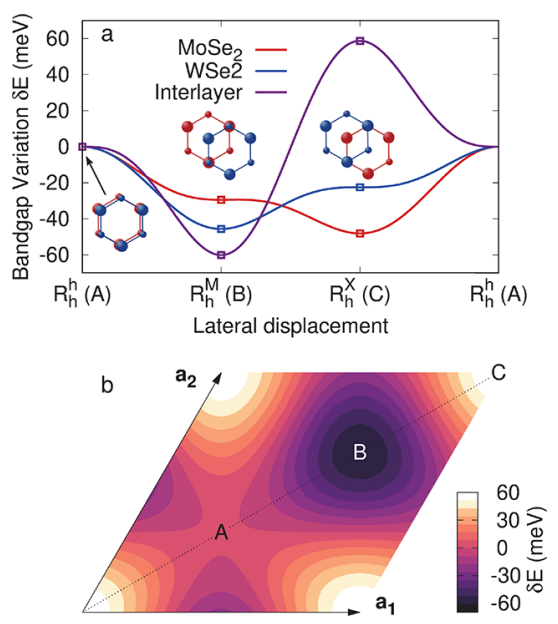

Figure 2. Moiré potential in the $\mathrm{MoSe}_{2} / \mathrm{WSe}_{2}$ heterostructure. (a) Band gap variation for the two intralayer transitions (red and blue) and the energetically lowest interlayer transition (purple) at the $\mathrm{K}$ point as a function of a lateral shift between the layers at zero twistangle. The dots show the energies calculated using $\mathrm{DFT}+\mathrm{G}_{0} \mathrm{~W}_{0}$, whereas the lines are extracted from a fitted microscopic model. (b) Interlayer band gap variation in a twisted heterostructure, where $\mathbf{a}_{i}$ values are the two fundamental translations of the moire superlattice. $\mathrm{A}, \mathrm{B}$, and $\mathrm{C}$ denote high-symmetry sites in the moire pattern, corresponding to local atomic registries specified in (a).

We find a triangular symmetry with a total minimum at the point denoted with $\mathrm{B}$ (local $\mathrm{R}_{h}^{M}$ stacking), a total maximum at $\mathrm{C}\left(\mathrm{R}_{h}^{X}\right)$, and an intermediate inflection point $\mathrm{A}\left(\mathrm{R}_{h}^{h}\right)$ (see Figure $2 \mathrm{~b}$ ). Based on the derived analytical model, we can now carry out a material-specific study of exciton characteristics in the moire potential of a twisted heterostructure. 
Excitonic Moiré Minibands. Due to the strong Coulomb interaction in 2D systems, the band-edge excitations in TMDs are governed by excitons, ${ }^{3}$ whose total energy is given by the band gap and the Coulomb binding energy. Consequently, a spatial band gap variation creates an effective potential influencing the exciton center-of-mass (CoM) motion. To account for the excitonic character of the elementary excitations in TMDs, we transform the Hamiltonian for conduction $(c)$ and valence band electrons $(v)$ into an exciton basis, $^{34,35} X_{\mathbf{Q}}^{\dagger}=\sum_{\mathbf{k}} \psi_{\mathbf{k}}^{*} c_{\mathbf{k}+\alpha \mathbf{Q}}^{\dagger} v_{\mathbf{k}-\beta \mathbf{Q}}$, with the ground-state exciton wave function $\psi_{\mathrm{k}}$, CoM momentum $\mathbf{Q}$ and mass coefficients $\alpha=m_{e} /\left(m_{e}+m_{h}\right), \beta=1-\alpha$. We thereby obtain an effective single-particle Hamiltonian:

$$
H=\sum_{\mu \mathbf{Q}} \mathcal{E}_{\mathbf{Q}}^{\mu} X_{\mu \mathbf{Q}}^{\dagger} X_{\mu \mathbf{Q}}+\sum_{\mu \mathbf{Q} \mathbf{q}} \mathcal{M}_{\mathbf{q}}^{\mu} X_{\mu \mathbf{Q}+\mathbf{q}}^{\dagger} X_{\mu \mathbf{Q}}
$$

containing the interaction-free part with the exciton dispersion $\mathcal{E}_{\mu \mathbf{Q}}$ and the effective exciton moire potential incorporating the spatial band gap variation (eq 1) convoluted with the exciton wave function (cf. Supporting Information). The index $\mu$ refers to different exciton states, composed either of electrons and holes within the same (intralayer exciton) or different layers (interlayer exciton). Note that we neglect the hybridization between intra- and interlayer exciton states as the electronic wave functions of valence and conduction bands at the $\mathrm{K}$ point are strongly localized in the two layers within the considered heterostructure. ${ }^{29,30}$ Equation 2 describes excitons moving in the effective periodic potential of the moire superlattice, analogous to electrons in a crystal. Hence, the spectrum of its eigenstates is given by a series of sub-bands $\zeta$ defined in the mini-Brillouin zone $(\mathrm{mBZ})$ spanned by the reciprocal lattice vectors of the moire pattern $\mathbf{g}_{n}$. We therefore diagonalize eq 2 for each exciton species via a zone-folding approach, yielding

$$
H=\sum_{\zeta \mathbf{Q}} E_{\mathbf{Q}}^{\zeta} Y_{\zeta \mathbf{Q}}^{\dagger} Y_{\zeta \mathbf{Q}}, \quad Y_{\zeta \mathbf{Q}}=\sum_{i, j} C_{i j \mathbf{Q}}^{\zeta} X_{\mathbf{Q}+i \mathbf{g}_{0}+j \mathbf{g}_{1}}
$$

In this framework, the emergence of moire excitons $Y$, analogous to Bloch waves of electrons in a crystal, can be understood as periodic superposition of CoM momenta, which has important consequences for optical selection rules of moiré excitons. $^{23}$

The calculated excitonic band structure for a twisted $\mathrm{MoSe}_{2} / \mathrm{WSe}_{2}$ bilayer is shown in Figure 3, exemplifying the $\mathrm{MoSe}_{2}$ intralayer exciton and the lower interlayer exciton of 1 and $3^{\circ}$, respectively. The dispersions are plotted along a highsymmetry path of the hexagonal $\mathrm{mBZ}$ with the center $\gamma$ and the edges $\kappa=\Delta K$. $^{18,21}$

At $1^{\circ}$, we find completely flat exciton bands for both interand intralayer excitons. Whereas flat bands for interlayer excitons have been already predicted in previous studies, ${ }^{24}$ intraband excitons were believed to have too shallow moire potentials to trap excitons. In this work, we find that GW corrections of the electronic band structure lead to significantly larger intralayer band gap variations in the range of $50 \mathrm{meV}$ (Figure 2), allowing for exciton localization at small angles. The appearance of nondispersed bands means that the corresponding exciton states have a vanishing group velocity and that hopping between neighboring moiré supercells is completely suppressed. Therefore, these states can be considered as moiré-localized zero-dimensional states analogous to quantum dots. Thereby the deeper interlayer exciton potential (Figure 2a) gives rise to several flat bands with large
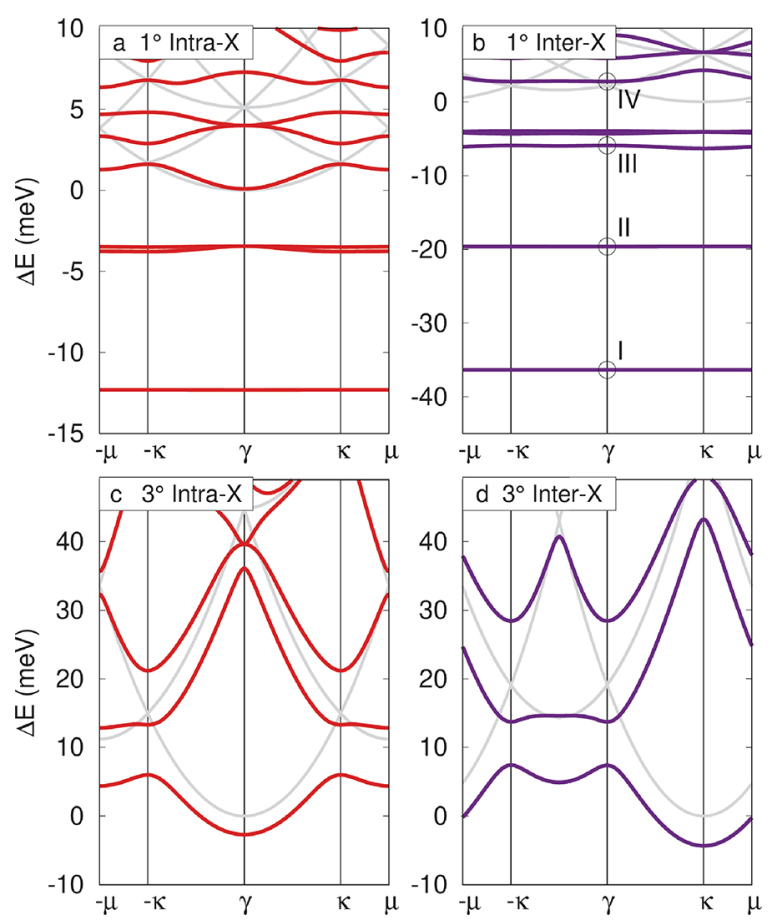

Figure 3. Moiré exciton minibands for the twisted $\mathrm{MoSe}_{2} / \mathrm{WSe}_{2}$ heterostructure. Whereas (a) and (b) show the $\mathrm{MoSe}_{2}$ intra- and the lowest interlayer exciton at $1^{\circ}$, respectively, (c) and (d) illustrate the corresponding bands at $3^{\circ}$. At $1^{\circ}$, both inter- and intralayer excitons exhibit flat, moire-trapped states, but only the deeper interlayer potential shows a whole series of localized states (denoted by I-III). For larger twist-angles, one finds almost parabolic bands close to a zone-folded free exciton dispersion (gray curves). Here, the free particle dispersion is only effectively modified at intersection points, where a strong mixing of different CoM momenta leads to avoided crossings that can be interpreted as standing waves at different sites of the moiré potential.

separations, whereas the shallower intralayer potential only allows one localized state and all excited states are spread over the whole crystal. These higher-order moire bands with a dispersive character can be interpreted as scattering states, that is, delocalized, free exciton waves with moiré-periodic amplitude modulations.

At $3^{\circ}$, all sub-bands are delocalized, exhibiting an almost parabolic dispersion close to the free particle situation (gray lines). This results from the shrinking of the moire supercell with increasing twist-angle. Consequently, the kinetic energy of the ground state, that is, the zero-point energy, increases with the twist-angle until the exciton starts to hop between neighboring moiré cells. However, the dispersion at $3^{\circ}$ still exhibits band gaps at intersection points of the zone-folded free-particle dispersion. Here, standing waves emerge from Bragg reflection at the moiré potential. The lowest interlayer exciton in Figure $3 \mathrm{~d}$ at the $\gamma$ point is a standing wave with large probability at B sites, whereas the second lowest state has the largest probability at A sites. The results shown in Figure 3 illustrate that the moiré exciton phase (including moiré localized or delocalized states) and consequently the excitonic transport properties can be widely tuned in TMD heterostructures, as the general character of the excitonic ground state is very sensitive to changes in the twist-angle-dependent moiré period. 
Moiré-Modified Light-Matter Coupling. The modulation of the exciton CoM momentum discussed above has a direct impact on the light-matter interaction in twisted heterostructures. Based on the transformation into a moire exciton basis (see Supporting Information), we calculate the absorption spectra ${ }^{36,37}$ of the twisted $\mathrm{MoSe}_{2}-\mathrm{WSe}_{2}$ heterostructure (Figure $4 a-d)$.
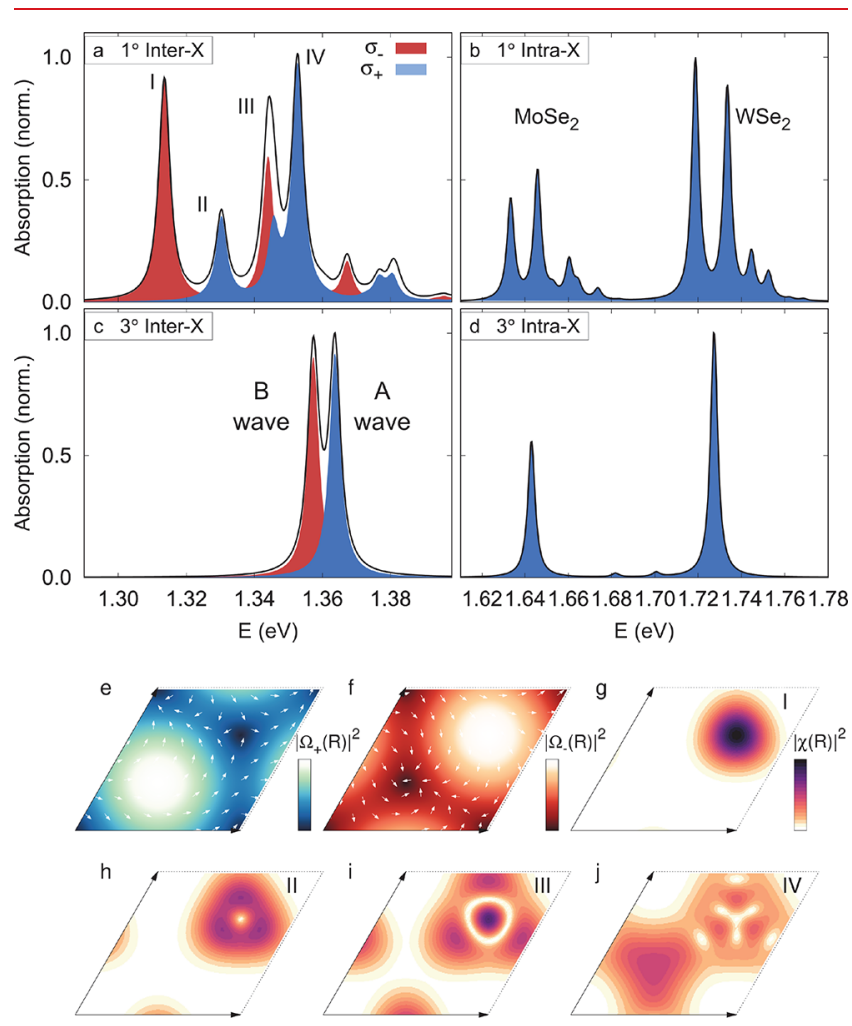

Figure 4. $(a-d)$ Moiré exciton absorption spectra at 1 and $3^{\circ}$. The moiré-induced mixing of exciton center-of-mass momenta gives rise to a splitting of the single exciton peak into a series of moire resonances at $1^{\circ}$ for both intra- as well as interlayer excitons. At $3^{\circ}$, the weak mixing leads to much smaller modifications of the absorption spectrum. Whereas intralayer resonances are fully $\sigma_{+}$polarized (blue), interlayer moiré excitons show an alternating $\sigma_{+}$and $\sigma_{-}$ polarization. (e,f) Spatial distribution of the oscillator strength for $\sigma_{+}$ and $\sigma_{-}$polarized light. The phase of the dipole matrix element is illustrated as a superimposed vector field. $(g-j)$ Exciton center-ofmass wave functions for states I-IV denoted in (a). For s-type states (I, III, and IV), the polarization of the corresponding absorption peaks results from the maximum of the wave function, whereas for $\mathrm{p}$ type states (II), the phase of the matrix element becomes crucial.

At $1^{\circ}$, we find multiple inter- and intralayer exciton peaks, whereas a perfectly aligned heterostructure would exhibit only one single resonance for each exciton species. To understand this moire phenomenon, we have to consider the momentum conservation for the light-matter interaction. In a regular semiconductor, only excitons with zero CoM momentum can be created by or decay into a photon as the latter has a negligible momentum. This selection rule is modified in a moiré superlattice, where Bragg reflection at the periodic potential can scatter excitons with nonzero momentum into the light cone. ${ }^{38}$ Consequently, all exciton minibands at the center of the $\mathrm{mBZ}$ ( $\gamma$ point) can, in principle, couple to photons. Their oscillator strength, $\Omega_{\zeta} \propto C_{\mathbf{Q}=0}^{\zeta}$, is determined by their projection onto the original bright state $(\mathbf{Q}=0)$; that is, the oscillator strength of the bright exciton is redistributed across all minibands according to the momentum spectrum of their wave function and is therefore mostly transferred to the lowest energy states with small kinetic energy. At $3^{\circ}$, we find only a single exciton resonance of the intralayer excitons, resembling the unperturbed $\mathbf{Q}=0$ exciton. In contrast, the interlayer exciton still shows two distinct resonances, resulting from the avoided crossing and the two low energy states corresponding to standing waves at $\mathrm{A}$ and $\mathrm{B}$ sites.

Although all intralayer exciton resonances are fully $\sigma_{+}$ polarized as in the monolayer case, we find alternating polarizations for the observed interlayer peaks. To explain this behavior microscopically, we show the optical matrix element for the interlayer electron-hole recombination for $\sigma_{+}$ and $\sigma_{-}$light in Figure 4e,f. The locally varying atomic alignment also has an impact on the optical selection rules for electronic transitions across both layers. Whereas coupling to $\sigma_{+}$light is strongest at A sites, it vanishes at B sites and the opposite is true for $\sigma_{-}$light. $^{24,26,38}$

We find that the CoM wave function for states I-III are localized at the potential minimum at the B site, whereas state IV is delocalized and has the largest probability at A sites (Figure $4 \mathrm{~g}-\mathrm{j}$ ). Comparing the overlap between wave functions and the spatial distribution of oscillator strength explains the polarization of peaks I, III $\left(\sigma_{-}\right)$, and IV $\left(\sigma_{+}\right)$. Interestingly, state II has p-type symmetry and should be dark because of its angular momentum (phase exp $i \phi$ ). However, the complex phase of the $\sigma_{+}$matrix element has the opposite winding number (cf. rotation of vector field around the B site) so that p-type states are bright in this spatial valley. Hence, the alternating series of polarizations of the interlayer absorption shown in Figure $4 a-d$ results from the ladder of alternating sand p-type states and/or probability maxima at A or B sites.

Twist-Tuning Moiré Excitonic Phases. Now, we investigate how the character and consequently the optical signatures of moire excitons evolve with the twist-angle (Figure 5 ). For both intra- and interlayer excitons, we find a multitude of absorption resonances, whose number gradually decreases with growing twist-angle, resulting from a decreased mixing between different moiré minibands. Therefore, the total oscillator strength of all moire resonances is conserved and given by the exciton transition dipole at $0^{\circ}$ twist. In order to understand the intriguing shifts of the appearing moire resonances, we have to distinguish between (i) moire-localized states and (ii) delocalized scattering states. For energy levels far below the unperturbed exciton ground states (dashed lines), the energy increases linearly with the twist-angle. These states correspond to zero-dimensional excitons deeply trapped in a moiré potential. With larger twist-angles, the moiré period decreases, which corresponds to a shorter confinement length. This leads to larger kinetic energies, so that the ground-state (zero-point) energy and the distance between different localized states increase ( $c f$. Figure $5 \mathrm{~b}$ for $\theta<1^{\circ}$ ). The closer a localized state gets to the free particle edge, the larger its CoM orbital becomes until it starts to overlap with neighboring cells and the exciton becomes delocalized. For the delocalized moiré resonances, we find a quadratic energy dependence on the twist-angle. This can be ascribed to the quadratic dispersion of the scattering states similar to the unperturbed system (cf. Figure $3 c, d$ ). With larger twist-angles, the size of the $\mathrm{mBZ}$ increases $(\kappa=\Delta K \propto \theta)$ and the intersection point of zone-folded branches with the light cone moves toward higher energies. 

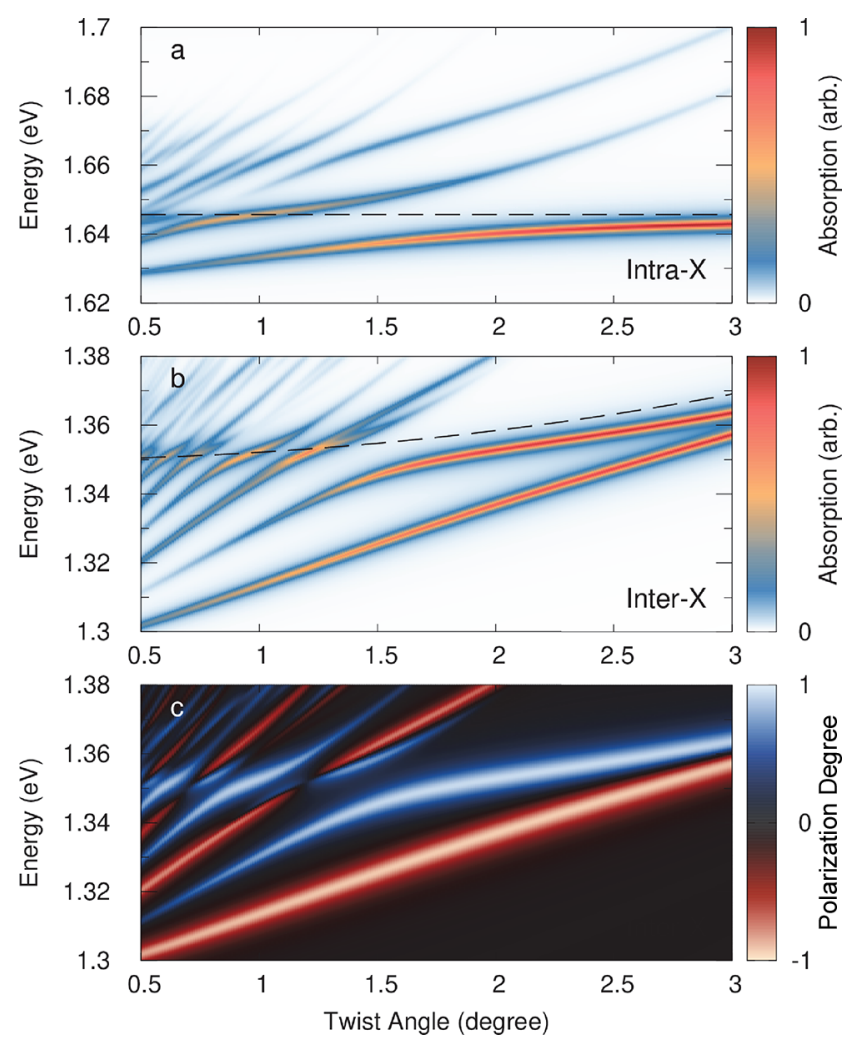

Figure 5. Absorption spectrum of (a) intralayer and (b) interlayer excitons as a continuous function of the twist-angle. Whereas all signals of the intralayer exciton are circularly polarized, the different interlayer exciton states show an alternating polarization, which is illustrated in (c). The twist-angle dependence of the multiple moire resonances varies for different exciton phases of moire-trapped and delocalized scattering states. For localized states with energies far below the free exciton (dashed lines), resonances shift linearly with the twist-angle. In contrast, moiré resonances stemming from scattering states (energies above or close to the dashed lines) have a quadratic twist-angle dependence reflecting the free-particle dispersion.

Note that the lowest intralayer exciton resonance stops shifting at a certain twist-angle, resembling the unperturbed bright exciton resonance at $\mathbf{Q}=0$. In contrast, the interlayer exciton resonance is further shifting upward in energy. This does not result from the periodic moire lattice but only reflects the indirect nature of the interlayer exciton in a twisted bilayer (cf. Figure 1). As valence and conduction bands are shifted away from each other, the minimum of the CoM dispersion is shifted away from the light cone (compare gray curves in Figure $3 b, d)$. Consequently, the bright state is moving up in energy in quadratic fashion, reflecting the dispersion of the free interlayer exciton. Finally, we find that the splitting between the two remaining interlayer exciton resonances at angles $>2.5^{\circ}$ (A and $\mathrm{B}$ wave resonance) decreases with increasing twistangle. This splitting is proportional to the effective moiré potential acting on the CoM coordinate of the exciton $\mathbf{R}$ (cf. eq 2), reading $\mathcal{M}^{\mu}(\mathbf{R})=\left\langle\mu\left|V^{c}(\mathbf{R}+\beta \mathbf{r})-V^{v}(\mathbf{R}-\alpha \mathbf{r})\right| \mu\right\rangle$. At large moire periods, the excitonic potential is given by the fluctuations of the band gap, $\mathcal{M} \approx V^{c}-V^{v}$. However, when the length scale of the moire period gets in the range of the exciton Bohr radius, the exciton does not interact with the potential like a point particle anymore. The excitonic potential is then given by a weighted average of the potential over the space occupied by the exciton. This explains the decreasing interlayer exciton splitting in Figure 5 for larger angles, as the exciton starts to occupy larger regions of the moire cell and thereby averages over potential minima and maxima.

\section{DISCUSSION}

The presented work provides a consistent microscopic framework to model the properties of moire excitons in twist-tunable van der Waals superlattices. In particular, it allows us to study transitions between different moiré exciton phases and their impact on the optical response of different heterostructures.

The four-fold interlayer exciton features shown in Figure 4a have recently been demonstrated in PL spectra. ${ }^{14}$ In particular, the measured peaks at $1^{\circ}$ exhibited a similar splitting in the range of $20 \mathrm{meV}$ and also showed alternating degrees of circular polarization, in excellent agreement with our results. Moreover, the predicted moire-induced splitting of intralayer excitons was experimentally shown in reflection contrast of $\mathrm{WS}_{2} / \mathrm{WSe}_{2}(\sim 50 \mathrm{meV})^{13}$ and photoluminescence in $\mathrm{MoSe}_{2} /$ $\mathrm{MoS}_{2}(\sim 11 \mathrm{meV})^{39}$ and was theoretically predicted for $\mathrm{MoS}_{2} /$ $\mathrm{WS}_{2}(\sim 10 \mathrm{meV}) .^{23}$ The intralayer double-peak structure for $\mathrm{MoSe}_{2} / \mathrm{WSe}_{2}$ with a splitting of about $10 \mathrm{meV}$ predicted in our work is in reasonable agreement with the above findings, considering the different material combination, but has not been observed experimentally yet.

Although the intralayer exciton features are directly accessible in absorption experiments, spatially indirect interlayer excitons are only visible in PL spectra due to their low oscillator strength. In PL, however, trions, ${ }^{40}$ defect-bound states, ${ }^{41}$ and other relaxation pathways can become important. In particular, momentum-indirect excitons involving holes at $\Gamma$ or electrons at $\Lambda$ might be energetically more favorable configurations then the direct $\mathrm{K}-\mathrm{K}$ excitons. ${ }^{29,42}$ However, moiré potentials with large periods compared to the unit cells cannot provide the momentum transfer necessary for an intervalley transition. Therefore, intervalley excitons still require the interaction with a phonon to recombine. ${ }^{43}$ In a systematic study varying excitation density, temperature, and applied electric/magnetic field, the above decay mechanism can potentially be disentangled. Moreover, future progress in stacking techniques might enable deterministic twist-angle studies with small step sizes of $0.1^{\circ}$, which would allow one to further experimentally verify the predicted twist-angle-dependent transition from a localized regime (linear shifts) to an unbound/scattering phase of excitons (parabolic shifts). The transition from moire-trapped to delocalized states was already observed in exciton diffusion measurements. ${ }^{44}$

Our work provides fundamental insights into exciton localization and light-matter coupling in twisted van der Waals heterostructures and will guide future experimental as well as theoretical studies in this growing field of research. In particular, the developed theoretical framework can be exploited to model exciton-exciton and exciton-phonon interactions governing the spatiotemporal dynamics of moiré excitons.

\section{ASSOCIATED CONTENT}

\section{Supporting Information}

The Supporting Information is available free of charge at https://pubs.acs.org/doi/10.1021/acs.nanolett.0c03019. 
Derivation of the effective continuum model for the moiré potential; details on the DFT and GW calculations and the extraction of model parameters; transformation of the Hamiltonian into moire exciton basis; derivation of the optical matrix elements and the absorption spectrum of moiré excitons; real space representation of center-of-mass wave functions (PDF)

\section{AUTHOR INFORMATION}

\section{Corresponding Author}

Samuel Brem - Department of Physics, Chalmers University of Technology, 41296 Gothenburg, Sweden; ○ orcid.org/00000001-8823-1302

\section{Authors \\ Christopher Linderälv - Department of Physics, Chalmers University of Technology, 41296 Gothenburg, Sweden \\ Paul Erhart - Department of Physics, Chalmers University of Technology, 41296 Gothenburg, Sweden; 이이이.org/0000- 0002-2516-6061 \\ Ermin Malic - Department of Physics, Chalmers University of Technology, 41296 Gothenburg, Sweden}

Complete contact information is available at:

https://pubs.acs.org/10.1021/acs.nanolett.0c03019

\section{Notes}

The authors declare no competing financial interest.

\section{ACKNOWLEDGMENTS}

We acknowledge funding from the Swedish Research Council (VR, Project No. 2018-00734), the European Unions Horizon 2020 research and innovation programme under Grant Agreement No. 881603 (Graphene Flagship), and the Knut and Alice Wallenberg Foundation (2014.0226).

\section{REFERENCES}

(1) He, K.; Kumar, N.; Zhao, L.; Wang, Z.; Mak, K. F.; Zhao, H.; Shan, J. Tightly bound excitons in monolayer $\mathrm{WSe}_{2}$. Phys. Rev. Lett. 2014, 113, 026803.

(2) Chernikov, A.; Berkelbach, T. C.; Hill, H. M.; Rigosi, A.; Li, Y.; Aslan, O. B.; Reichman, D. R.; Hybertsen, M. S.; Heinz, T. F. Exciton binding energy and nonhydrogenic Rydberg series in monolayer $\mathrm{WS}_{2}$. Phys. Rev. Lett. 2014, 113, 076802.

(3) Wang, G.; Chernikov, A.; Glazov, M. M.; Heinz, T. F.; Marie, X.; Amand, T.; Urbaszek, B. Colloquium: Excitons in atomically thin transition metal dichalcogenides. Rev. Mod. Phys. 2018, 90, 021001.

(4) Mueller, T.; Malic, E. Exciton physics and device application of two-dimensional transition metal dichalcogenide semiconductors. $n p j$ 2D Materials and Applications 2018, 2, 29.

(5) Niehues, I.; Schmidt, R.; Drüppel, M.; Marauhn, P.; Christiansen, D.; Selig, M.; Berghäuser, G.; Wigger, D.; Schneider, R.; Braasch, L.; et al. Strain control of exciton-phonon coupling in atomically thin semiconductors. Nano Lett. 2018, 18, 1751-1757.

(6) Aslan, O. B.; Deng, M.; Heinz, T. F. Strain tuning of excitons in monolayer $\mathrm{WSe}_{2}$. Phys. Rev. B: Condens. Matter Mater. Phys. 2018, 98, 115308.

(7) Latini, S.; Olsen, T.; Thygesen, K. S. Excitons in van der Waals heterostructures: The important role of dielectric screening. Phys. Rev. B: Condens. Matter Mater. Phys. 2015, 92, 245123.

(8) Steinleitner, P.; Merkl, P.; Graf, A.; Nagler, P.; Watanabe, K.; Taniguchi, T.; Zipfel, J.; Schüller, C.; Korn, T.; Chernikov, A.; et al. Dielectric engineering of electronic correlations in a van der Waals heterostructure. Nano Lett. 2018, 18, 1402-1409.

(9) Raja, A.; Waldecker, L.; Zipfel, J.; Cho, Y.; Brem, S.; Ziegler, J. D.; Kulig, M.; Taniguchi, T.; Watanabe, K.; Malic, E.; et al. Dielectric disorder in two-dimensional materials. Nat. Nanotechnol. 2019, 14, 832-837.

(10) Rivera, P.; Schaibley, J. R.; Jones, A. M.; Ross, J. S.; Wu, S.; Aivazian, G.; Klement, P.; Seyler, K.; Clark, G.; Ghimire, N. J.; Yan, J.; Mandrus, D. G.; Yao, W.; Xu, X. Observation of long-lived interlayer excitons in monolayer $\mathrm{MoSe}_{2}-\mathrm{WSe}_{2}$ heterostructures. Nat. Commun. 2015, 6, 6242.

(11) Miller, B.; Steinhoff, A.; Pano, B.; Klein, J.; Jahnke, F.; Holleitner, A.; Wurstbauer, U. Long-lived direct and indirect interlayer excitons in van der Waals heterostructures. Nano Lett. 2017, 17, 5229-5237.

(12) Merkl, P.; Mooshammer, F.; Steinleitner, P.; Girnghuber, A.; Lin, K.-Q.; Nagler, P.; Holler, J.; Schüller, C.; Lupton, J. M.; Korn, T.; et al. Ultrafast transition between exciton phases in van der Waals heterostructures. Nat. Mater. 2019, 18, 691-696.

(13) Jin, C.; Regan, E. C.; Yan, A.; Iqbal Bakti Utama, M.; Wang, D.; Zhao, S.; Qin, Y.; Yang, S.; Zheng, Z.; Shi, S.; Watanabe, K.; Taniguchi, T.; Tongay, S.; Zettl, A.; Wang, F. Observation of moiré excitons in $\mathrm{WSe}_{2} / \mathrm{WS}_{2}$ heterostructure superlattices. Nature 2019, $567,76-80$.

(14) Tran, K.; Moody, G.; Wu, F.; Lu, X.; Choi, J.; Kim, K.; Rai, A.; Sanchez, D. A.; Quan, J.; Singh, A.; et al. Evidence for moiré excitons in van der Waals heterostructures. Nature 2019, 567, 71-75.

(15) Seyler, K. L.; Rivera, P.; Yu, H.; Wilson, N. P.; Ray, E. L.; Mandrus, D. G.; Yan, J.; Yao, W.; Xu, X. Signatures of moiré-trapped valley excitons in $\mathrm{MoSe}_{2} / \mathrm{WSe}_{2}$ heterobilayers. Nature 2019, 567, 6670.

(16) Alexeev, E. M.; Ruiz-Tijerina, D. A.; Danovich, M.; Hamer, M. J.; Terry, D. J.; Nayak, P. K.; Ahn, S.; Pak, S.; Lee, J.; Sohn, J. I.; et al. Resonantly hybridized excitons in moiré superlattices in van der Waals heterostructures. Nature 2019, 567, 81-86.

(17) Wang, Y.; Wang, Z.; Yao, W.; Liu, G.-B.; Yu, H. Interlayer coupling in commensurate and incommensurate bilayer structures of transition-metal dichalcogenides. Phys. Rev. B: Condens. Matter Mater. Phys. 2017, 95, 115429.

(18) Ruiz-Tijerina, D. A.; Fal'ko, V. I. Interlayer hybridization and moiré superlattice minibands for electrons and excitons in heterobilayers of transition-metal dichalcogenides. Phys. Rev. B: Condens. Matter Mater. Phys. 2019, 99, 125424.

(19) Merkl, P.; Mooshammer, F.; Brem, S.; Girnghuber, A.; Lin, K. Q.; Weigl, L.; Liebich, M.; Yong, C.-K.; Gillen, R.; Maultzsch, J.; Lupton, J. M.; Malic, E.; Huber, R. Twist-tailoring Coulomb correlations in van der Waals homobilayers. Nat. Commun. 2020, $11,2167$.

(20) Kiemle, J.; Sigger, F.; Lorke, M.; Miller, B.; Watanabe, K.; Taniguchi, T.; Holleitner, A.; Wurstbauer, U. Control of the orbital character of indirect excitons in $\mathrm{MoS}_{2} / \mathrm{WS}_{2}$ heterobilayers. Phys. Rev. B: Condens. Matter Mater. Phys. 2020, 101, 121404.

(21) Brem, S.; Lin, K.-Q.; Gillen, R.; Bauer, J. M.; Maultzsch, J.; Lupton, J. M.; Malic, E. Hybridized intervalley moiré excitons and flat bands in twisted $\mathrm{WSe}_{2}$ bilayers. Nanoscale 2020, 12, 11088.

(22) Zhang, C.; Chuu, C.-P.; Ren, X.; Li, M.-Y.; Li, L.-J.; Jin, C.; Chou, M.-Y.; Shih, C.-K. Interlayer couplings, Moiré patterns, and 2D electronic superlattices in $\mathrm{MoS}_{2} / \mathrm{WSe}_{2}$ hetero-bilayers. Science Advances 2017, 3, No. e1601459.

(23) Wu, F.; Lovorn, T.; MacDonald, A. H. Topological exciton bands in moiré heterojunctions. Phys. Rev. Lett. 2017, 118, 147401.

(24) Wu, F.; Lovorn, T.; MacDonald, A. Theory of optical absorption by interlayer excitons in transition metal dichalcogenide heterobilayers. Phys. Rev. B: Condens. Matter Mater. Phys. 2018, 97, 035306.

(25) Wu, F.; Lovorn, T.; Tutuc, E.; MacDonald, A. H. Hubbard model physics in transition metal dichalcogenide moiré bands. Phys. Rev. Lett. 2018, 121, 026402.

(26) Yu, H.; Liu, G.-B.; Tang, J.; Xu, X.; Yao, W. Moiré excitons: From programmable quantum emitter arrays to spin-orbit-coupled artificial lattices. Science Advances 2017, 3, No. e1701696. 
(27) Liu, K.; Zhang, L.; Cao, T.; Jin, C.; Qiu, D.; Zhou, Q.; Zettl, A.; Yang, P.; Louie, S. G.; Wang, F. Evolution of interlayer coupling in twisted molybdenum disulfide bilayers. Nat. Commun. 2014, 5, 4966.

(28) Naik, M. H.; Jain, M. Ultraflatbands and shear solitons in moiré patterns of twisted bilayer transition metal dichalcogenides. Phys. Rev. Lett. 2018, 121, 266401.

(29) Gillen, R.; Maultzsch, J. Interlayer excitons in $\mathrm{MoSe}_{2} / \mathrm{WSe}_{2}$ heterostructures from first principles. Phys. Rev. B: Condens. Matter Mater. Phys. 2018, 97, 165306.

(30) Lu, X.; Li, X.; Yang, L. Modulated interlayer exciton properties in a two-dimensional moiré crystal. Phys. Rev. B: Condens. Matter Mater. Phys. 2019, 100, 155416.

(31) Guo, H.; Zhang, X.; Lu, G. Shedding Light on Moire Excitons: A First-Principles Perspective. arXiv:2005.00612 2020; https://arxiv. org/abs/2005.0612.

(32) Cao, Y.; Fatemi, V.; Fang, S.; Watanabe, K.; Taniguchi, T.; Kaxiras, E.; Jarillo-Herrero, P. Unconventional superconductivity in magic-angle graphene superlattices. Nature 2018, 556, 43-50.

(33) Bistritzer, R.; MacDonald, A. H. Moiré bands in twisted double-layer graphene. Proc. Natl. Acad. Sci. U. S. A. 2011, 108, $12233-12237$.

(34) Haug, H.; Schmitt-Rink, S. Electron theory of the optical properties of laser-excited semiconductors. Prog. Quantum Electron. 1984, 9, 3-100.

(35) Katsch, F.; Selig, M.; Carmele, A.; Knorr, A. Theory of Exciton-Exciton Interactions in Monolayer Transition Metal Dichalcogenides. Phys. Status Solidi B 2018, 255, 1800185.

(36) Kira, M.; Koch, S. Many-body correlations and excitonic effects in semiconductor spectroscopy. Prog. Quantum Electron. 2006, 30, $155-296$.

(37) Koch, S.; Kira, M.; Khitrova, G.; Gibbs, H. Semiconductor excitons in new light. Nat. Mater. 2006, 5, 523-531.

(38) Yu, H.; Wang, Y.; Tong, Q.; Xu, X.; Yao, W. Anomalous light cones and valley optical selection rules of interlayer excitons in twisted heterobilayers. Phys. Rev. Lett. 2015, 115, 187002.

(39) Zhang, N.; Surrente, A.; Baranowski, M.; Maude, D. K.; Gant, P.; Castellanos-Gomez, A.; Plochocka, P. Moiré intralayer excitons in a $\mathrm{MoSe}_{2} / \mathrm{MoS}_{2}$ heterostructure. Nano Lett. 2018, 18, 7651-7657.

(40) Bellus, M. Z.; Ceballos, F.; Chiu, H.-Y.; Zhao, H. Tightly bound trions in transition metal dichalcogenide heterostructures. ACS Nano 2015, 9, 6459-6464.

(41) Vialla, F.; Danovich, M.; Ruiz-Tijerina, D. A.; Massicotte, M.; Schmidt, P.; Taniguchi, T.; Watanabe, K.; Hunt, R. J.; Szyniszewski, M.; Drummond, N. D.; et al. Tuning of impurity-bound interlayer complexes in a van der Waals heterobilayer. 2D Mater. 2019, 6, 035032.

(42) Kunstmann, J.; Mooshammer, F.; Nagler, P.; Chaves, A.; Stein, F.; Paradiso, N.; Plechinger, G.; Strunk, C.; Schüller, C.; Seifert, G.; et al. Momentum-space indirect interlayer excitons in transition-metal dichalcogenide van der Waals heterostructures. Nat. Phys. 2018, 14, 801-805.

(43) Brem, S.; Ekman, A.; Christiansen, D.; Katsch, F.; Selig, M.; Robert, C.; Marie, X.; Urbaszek, B.; Knorr, A.; Malic, E. Phononassisted photoluminescence from indirect excitons in monolayers of transition-metal dichalcogenides. Nano Lett. 2020, 20, 2849-2856.

(44) Choi, J.; Hsu, W.-T.; Lu, L.-S.; Sun, L.; Cheng, H.-Y.; Lee, M.H.; Quan, J.; Tran, K.; Wang, C.-Y.; Staab, M. Moiré Potential Impedes Interlayer Exciton Diffusion in van der Waals Heterostructures. arXiv:1912.11101 2020; https://arxiv.org/abs/1912.1110. 